E lectromyography (EMG), the recording of electrical activity in muscle, should be regarded as an extension of the clinical examination. It can distinguish myopathic from neurogenic muscle wasting and weakness. It can detect abnormalities such as chronic denervation or fasciculations in clinically normal muscle. It can, by determining the distribution of neurogenic abnormalities, differentiate focal nerve, plexus, or radicular pathology; and it can provide supportive evidence of the pathophysiology of peripheral neuropathy, either axonal degeneration or demyelination. EMG is an obligatory investigation in motor neurone disease to demonstrate the widespread denervation and fasciculation required for secure diagnosis.

\title{
EMG METHODOLOGY
}

Recordings are made with a disposable concentric needle electrode inserted into the muscle. A fine wire in the axis of the needle is insulated from the shaft, the end of the needle being cut at an acute angle. The area of the recording surface determines the volume of muscle that the needle can "see". Conventional EMG needles record from a hemisphere of radius of about $1 \mathrm{~mm}$. Within this volume there are some 100 muscle fibres. The many hundreds of muscle fibres belonging to one motor unit are distributed widely throughout the cross section of the muscle and, therefore, within the pick-up region of the needle there may be just 4-6 fibres of a single motor unit. Analysis of the waveforms and firing rates of single motor or multiple motor units can give diagnostic information.

Electromyographers are skilled at interpreting both the appearance of muscle activity and the sound of the activity transmitted through a loud speaker. Normal resting muscle is silent. Patients often have difficulty completely relaxing a muscle. The motor unit activity associated with incomplete relaxation is distinguished from abnormal spontaneous activity by its rhythmicity. Motor units when first recruited or on the point of being de-recruited fire regularly at 6-10 spikes per second. Voluntary firing caused by incomplete relaxation can often be silenced by passively changing the posture of the limb or by slight activation of the antagonist. Voluntary motor units never fire as single isolated discharges, a useful point in distinguishing them from fasciculations.

\section{SPONTANEOUS ACTIVITY}

\section{Fibrillation, positive waves, and complex repetitive discharges}

After an acute nerve transection, nerve fibres degenerate from the site of the lesion distally. Muscle fibres themselves remain viable but after a period of 7-10 days become supersensitive and fibrillations will be detectable. Acutely denervated muscle fibres have acetylcholine receptors over the whole of the muscle fibre membrane rather than these being limited to the neuromuscular junction. The effect is to make the fibre supersensitive with the result that it discharges spontaneously. This is detected by the EMG needle as a single fibre discharge or fibrillation (fig 1A). Fibrillation is not visible through the skin and is an electrical sign not a clinical sign. Positive sharp waves have the same origin as fibrillation and have the same significance. They arise when the needle tip damages a fibre and spontaneous action potentials propagate up to the needle tip and then are extinguished. Fibrillation may persist for many months after a nerve lesion. Any nerve lesion, complete or partial, from the spinal motor neurone to the intramuscular nerve branches can give rise to fibrillation. Fibrillations are not found exclusively in neurogenic disease, however; they also occur in inflammatory and dystrophic muscle disease.

Complex repetitive discharges begin and end abruptly, may persist for several minutes (or until the electromyographer gets bored), have a constant frequency between $1-100 \mathrm{~Hz}$, and consist of a stereotyped group of single fibre potentials. The minimal jitter between potentials suggests that they arise by ephaptic transmission between adjacent fibres. They occur predominantly in neurogenic disease.

Correspondence to: Department of Clints, Neurophysiology, King's College Hospital, 'Denmark Hill, London SE5 9RS, UK; kerry.mills1@kingsch.nhs.uk 


\section{Fasciculation}

Fasciculations arise from the discharge of part or the whole of a single motor unit (fig $1 \mathrm{C}$ ). They are larger and more complex than fibrillation potentials. Fasciculations are isolated discharges that recur at irregular intervals, usually in the order of several seconds. Fasciculations near the surface of a muscle may be visible at the skin; those deep within the muscle and detected by an EMG needle are not. Fasciculations are not under voluntary control, a useful point in distinguishing them from motor units discharging due to poor relaxation. Fasciculations probably arise within the fine terminal arborisation of a single motor axon within the muscle. It has been shown, for example, that there may be more than one generator of a single fasciculation as evidenced by subcomponents of the potential occurring in a different order on different discharges. Fasciculations occur in motor neurone diseases, other neurogenic diseases such as radiculopathy and neuropathy, thyroid disease, and peripheral nerve hyperexcitability syndromes, and may be benign. The significance of fasciculations is judged by the company they keep in the muscle; benign fasciculations are not accompanied by denervation changes but malign fasciculations usually are.

\section{Myotonia}

Clinical myotonia is accompanied by myotonic discharges on EMG (fig 1D); once heard, never forgotten. Discharges are provoked by needle movement, tapping the muscle and after a short voluntary contraction, and vary in frequency and amplitude producing the characteristic "dive bomber" sound. They occur in muscle fibre membrane channelopathies, including dystrophia myotonica, congenital myotonias, proximal myotonic dystrophy, and hypokalaemic periodic paralysis.

\section{Neuromyotonia}

Neuromyotonia arises from hyperexcitability of single peripheral motor axons either before or after they branch within the muscle, and is characterised by doublet, triplet, or short bursts of high frequency discharge (fig 1B) of motor unit, partial motor unit, or single fibre potentials which produce a "ping" sound from the EMG machine. Neuromyotonia occurs in autoimmune anti-voltage gated potassium nerve axon channelopathy and Morvan's syndrome.

\section{Myokymia}

This is the regular or irregular discharge of groups of motor units producing the clinical appearance of flickering in the muscles. It occurs in a wide variety of conditions, notably with brain stem neoplasms or demyelination and subclinically in episodic ataxia type I. EMG is useful in distinguishing myokymia from fasciculation, neuromyotonia and myoclonus.

\section{MOTOR UNIT RECRUITMENT}

Patients requested to activate minimally a muscle are usually able to recruit a single or a few motor units. Motor units have a recruitment frequency of $6-10 \mathrm{~Hz}$ and are recognisable by the constancy of the waveform of each discharge (fig 2). A number of parameters of motor unit potentials can be measured; this is achieved by triggering the sweep of an EMG machine by the potential crossing of a threshold and displaying the potential in its entirety. Amplitude, duration, number of phases (changes in direction), and firing rate are useful. When the patient makes a stronger contraction, more motor units are recruited and potentials overlap and interfere with each other; hence this is sometimes referred to as the interference pattern. Recruitment pattern is now the preferred term. At maximal voluntary activation, a full recruitment pattern is produced which fills the baseline. A reduced pattern occurs when despite maximal activation, which of course must be assessed clinically, areas of baseline without activity can still be seen.

\section{Reduced recruitment}

A clinical judgement must be made by the electromyographer on the significance of reduced recruitment. Muscle contraction limited by pain, a patient just not trying, an upper motor neurone lesion, motor conduction block, or chronic partial denervation may all cause a reduced recruitment pattern. The last is distinguished, most obviously by the sound of the activity, by the presence of large motor units firing at high
A

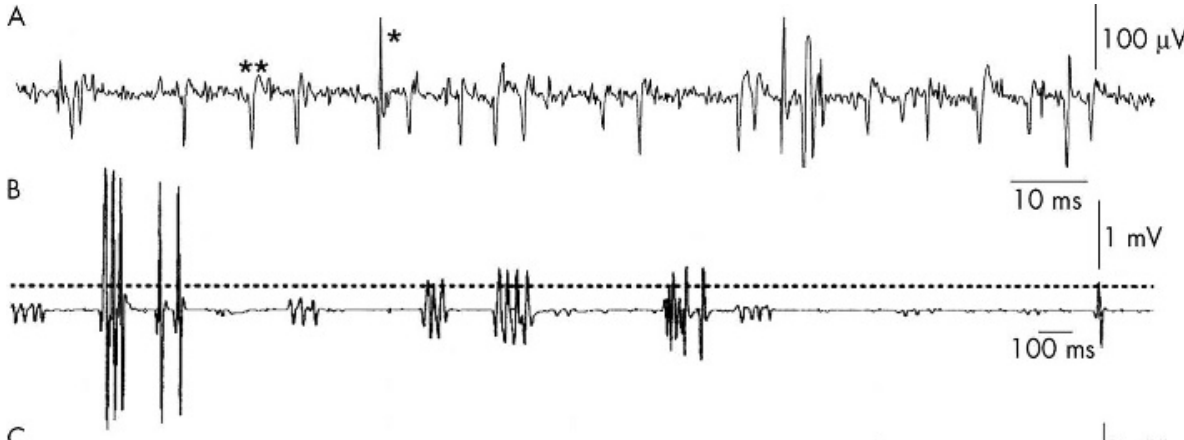

C

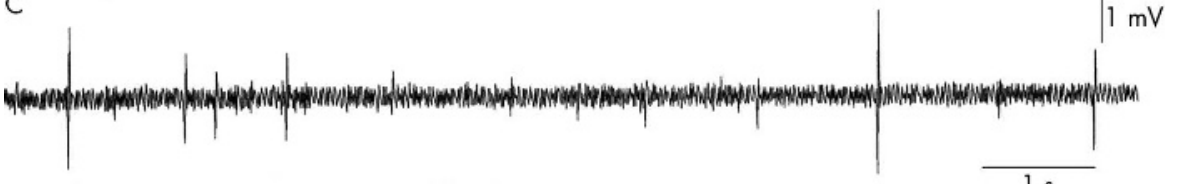

$1 \mathrm{~s}$
Figure 1 Abnormal spontaneous activity. (A) Fibrillations (*) and positive sharp waves $\left(^{* *}\right)$ in an acutely denervated hand muscle. (B) Single, doublet, triplet, and multiplet motor unit neuromyotonic discharges. Bursts of discharge are irregular in frequency and the intra-burst frequency of discharge is up to $200 \mathrm{~Hz}$. (C) Fasciculations in the tongue in a patient with amyotrophic lateral sclerosis. The single discharges are irregular and occur on a background of ongoing EMG activity caused by poor relaxation. (D) Myotonic discharges in a patient with dystrophia myotonica. There is a characteristic waxing and waning in frequency. 


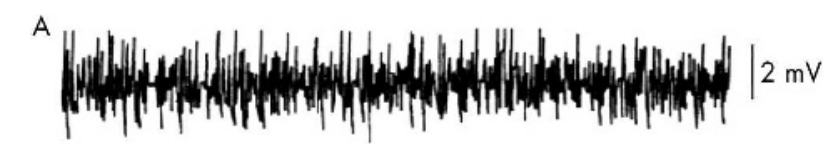

ii34
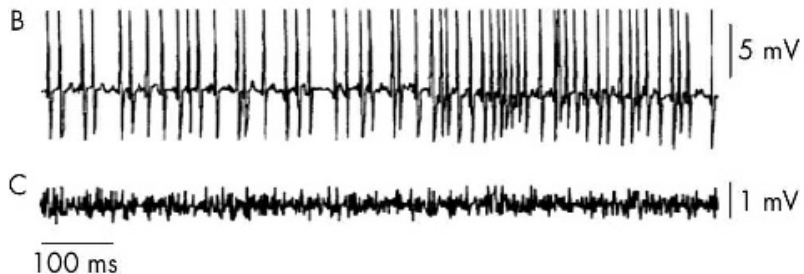

Figure 2 Recruitment patterns during maximal voluntary contraction of the deltoid muscle in (A) a healthy subject, (B) a patient with spinal muscular atrophy, and (C) a patient with polymyositis. Note the different amplitude calibrations.

rates. A fluctuating voluntary effort is accompanied by sporadic recruitment of motor units and is easily recognised by an experienced electromyographer.

\section{Early recruitment}

In primary muscle disease, recruitment of motor units is described as early-that is, early in the progressive recruitment of motor units with increasing force of contraction. Even when the patient only produces a low force contraction, the recruitment pattern is full. In florid primary muscle disease, early recruited motor units combine to produce a characteristic high frequency sound on the EMG machine.

\section{Motor unit potentials}

By isolating the discharge of single motor units by triggering and delaying the display, parameters of the motor unit potential can be measured. Motor unit parameters vary with the muscle being examined. Amplitude, duration, and number of phases may be measured. Chronic re-innervation is associated with high amplitude, long duration motor unit potentials with a normal number of phases. As a rule of thumb, motor unit potentials are less than $2 \mathrm{mV}$ in amplitude, and have a duration of 10-5 ms with 3-4 phases. In chronic partial denervation where intramuscular sprouting and re-innervation has occurred, amplitudes may be 10$20 \mathrm{mV}$ and durations $20-30 \mathrm{~ms}$. In primary muscle disease, motor unit potentials are small and of short duration; typical values would be $0.5 \mathrm{mV}$ and 5-10 ms. Polyphasicity can be seen in a wide number of situations, but muscle disease and the early stages of re-innervation are the most common.

\section{Tremor}

Simple surface EMG recordings can easily be used to measure the frequency of tremor. A 4 second, say, length of record is taken and the number of tremor bursts counted. In one condition, primary orthostatic tremor, the findings are so characteristic as to be diagnostic. If recordings are made from leg muscles, on standing, a highly synchronised tremor at a frequency of 14-18 Hz is seen; this disappears on sitting.

A number of clinical scenarios in which EMG is of proven use are now presented.

\section{PERIPHERAL NEUROPATHY}

By and large, neurophysiological diagnosis of peripheral neuropathy is achieved by nerve conduction studies which can differentiate the two major types-axonal degeneration and demyelinating. EMG, however, can give additional diagnostic or prognostic information. In acute inflammatory demyelinating polyneuropathy (Guillain-Barré syndrome (GBS)), if as is usually the case, the predominant cause of weakness is conduction block, EMG shows merely a reduced recruitment pattern of motor units of normal appearance. If, however, there has been secondary axonal degeneration, or if the case is an example of the rarer axonal form of GBS, then fibrillations are seen indicating axonal loss. Clearly, later in the evolution of GBS, the finding of signs of axonal loss imply a rather poorer prognosis for the recovery of function. Similarly, in chronic demyelinating neuropathy-for example, chronic inflammatory demyelinating polyradiculoneuropathy (CIDP) - axonal loss, as evidenced by fibrillations and signs of chronic reinnervation on EMG, indicate a less than favourable prognosis for recovery of function. Vasculitic neuropathies usually produce pain, and nerve conduction studies and EMG are again used in combination to demonstrate acute, usually single, nerve lesions. In the acute stage of a vasculitic nerve lesion, the nerve remains excitable distal to the lesion and it appears on nerve conduction studies as conduction block. Only after the nerve has begun to degenerate do the axons become inexcitable distal to the lesion and EMG signs of acute denervation appear. Axonal degeneration type peripheral neuropathies often produce fibrillations in distal foot muscles. Indeed, occasionally this can be the only sign of a neuropathy. Caution is required, however, as even in healthy elderly subjects fibrillation can be found in small foot muscles, presumably as a result of the traumas of everyday life.

\section{ENTRAPMENT NEUROPATHY}

Again, in most of the common entrapments, carpal tunnel syndrome, ulnar nerve lesions at the elbow, and common peroneal nerve lesions at the fibular head, nerve conduction studies are the principal diagnostic techniques. A number of less common entrapments, however, are not amenable to diagnosis with nerve conduction measurements, and localisation depends on an EMG demonstration of a particular distribution of abnormality. For example, median nerve lesions in the upper arm, those involving the anterior interosseous branch, and the common type at the wrist can be distinguished by EMG of the pronator teres (from the highest branch), finger and wrist flexors, and abductor pollicis brevis. The anterior interosseous branch is purely motor and therefore sensory action potentials from the median innervated fingers are normal. Similarly, the differentiation of radial from posterior interosseous nerve lesions rests on the finding of EMG abnormalities in triceps and brachioradialis in the former but not in the latter. Thirdly, although the sciatic nerve can be stimulated electrically, nerve conduction is difficult to interpret and EMG is the only means to differentiate sciatic lesions from S1 lesions by showing in the latter EMG signs of denervation in the muscles innervated by the superior and inferior gluteal nerves (glutei).

\section{BRACHIAL PLEXUS LESIONS}

Upper limb muscles can be sampled by EMG to determine the distribution of dennervation changes and determine whether they accord to the root, cord, trunk, and nerve innervation to provide localisation of brachial plexus lesions (table 1). The same logical principles as are employed in clinical examination pertain-except that EMG has the added bonus of showing abnormality in clinically unaffected muscles. For 
Table 1 Innervation of upper limb muscles

\begin{tabular}{lllll}
\hline Muscle & Nerve & Cord & Trunk & Roots \\
\hline Supraspinatus & Suprascapular & - & Upper & C4,C5, C6 \\
Deltoid & Axillary & Posterior & Upper & C5, C6 \\
Biceps & Musculo-cutaneous & Lateral & Upper & C5, C6 \\
Brachioradialis & Radial & Posterior & Upper & C5, C6 \\
FCR & Median & Lateral and medial & Upper and middle & C6, C7, C8 \\
EDC & Radial & Posterior & Lower and middle & C7, C8, T1 \\
Triceps & Radial & Posterior & Upper & C7, C8, T1 \\
APB & Median & Medial & Lower & C7, C8 \\
FCU & Ulnar & Medial & Lower & C8, T1 \\
FDI & Ulnar & Medial & Lower & C8, T1 \\
\hline
\end{tabular}

APB, abductor pollicis brevis; EDC, extensor digitorum communis; FCR, flexor carpi radialis; FCU, flexor carpi ulnaris; FDI, first dorsal interosseous.

example, a posterior cord lesion of the brachial plexus would show denervation in deltoid, triceps and EDC, but biceps would be normal.

\section{MOTOR NEURONE DISEASES}

In the absence of a reliable marker for idiopathic amyotrophic lateral sclerosis (ALS), EMG forms the cornerstone of diagnosis. Conditions that simulate ALS must be eliminated and there must be clear evidence of widespread denervation before a secure diagnosis can be entertained. The conditions simulating ALS include cervical myelopathy with radiculopathy which can easily produce upper motor neurone features in the legs with lower motor neurone features in the arms, and multifocal motor neuropathy with conduction block in which reflexes are usually retained and fasciculations may be evident. In the former, it is clearly mandatory to show evidence of a lower motor neurone component in muscles innervated by nerves emerging above the foramen magnum. Thus trapezius, sternomastoid, and the tongue are examined for evidence of fasciculation and/or chronic partial denervation. The tongue is particularly important because of its frequent involvement in ALS. The submental approach to the tongue is preferred and the needle position adjusted or "tuned" with a slight activation of the tongue by asking the patient to press the tongue gently against the bottom teeth. Complete relaxation of the tongue is rarely achieved, but fasciculations can sometimes be recognised by the fact that they occur as an isolated discharge and are much larger than the ongoing voluntary activity (fig $1 \mathrm{C}$ ). A partially denervated tongue often produces the characteristic sound of high firing rate motor units even though the amplitudes may not be excessively large. In multifocal motor neuropathy, conduction block, usually in the innervation of a small hand muscle and usually not at a common entrapment site, is demonstrated by motor nerve conduction studies. Fasciculations in multifocal motor neuropathy (MMN) are focal and occur only in muscles showing conduction block; indeed, they are believed to arise at the focal area of block. Muscles that are clinically unaffected in MMN show no EMG abnormality, in contrast to ALS where clinically unaffected muscles commonly show fasciculations and partial denervation.

The best approach to ALS is to show normal motor and sensory nerve conduction in the arms and legs and then to use EMG to demonstrate widespread fasciculations and chronic partial denervation in all four limbs and the tongue. In addition, transcranial magnetic stimulation can be used to document the existence of an upper motor neurone lesion; this is especially useful in those cases of ALS presenting as an initially pure lower motor neurone syndrome (progressive muscular atrophy).

More chronic motor neurone diseases, either inherited (for example, spinal muscular atrophies) or acquired (for example, poliomyelitis), are characterised by pronounced chronic partial denervation changes evidenced by large (15$20 \mathrm{mV}$ ) simple shaped motor unit potentials with little if any fibrillation.

The two above examples typify the way prognostic estimates are made in ALS. The balance between acute denervation and the effects of re-innervation influences the degree to which acute (fibrillation) and chronic (large motor units) changes are found on EMG.

\section{PRIMARY MUSCLE DISEASE}

In general, the EMG in primary muscle disease of any origin is similar and making distinctions between, say, hereditary and acquired myopathy is difficult. Motor unit potentials are small and spiky and the recruitment pattern becomes full with just a small contraction. In inflammatory muscle disease where there is active degeneration of muscle fibres, fibrillations may be seen, but this is not universal or specific. In muscle diseases which show large variation in fibre diameter, such as the muscular dystrophies, there may be large motor units on a background of small spiky units, presumably arising from large diameter fibres. In cases of Lambert-Eaton myasthenic syndrome (LEMS), the EMG can look myopathic because so many muscle fibres have neuromuscular block; the EMG becomes more normal if the patient is able to maintain a voluntary contraction. In any case where a myopathic EMG is found, LEMS should be considered and should be investigated by measuring the amplitude of a compound muscle action potential evoked by nerve stimulation before and after exercise.

\section{REFERENCES}

1 Aminoff, MJ. Electromyography in clinical practice. 3rd ed. New York: Churchill Livingstone, 1997:1-630.

2 Brown WF, Bolton CF, eds. Clinical electromyography. Boston: ButterworthHeinemann, 1993:1-810.

3 Delisa JA, Lee HJ. Manual of nerve conduction and surface anatomy for needle electromyography. Philadelphia: Lippincott, Williams and Wilkins, 2004: 1-301.

4 Dumitru D, Amata, A, Zwarts M. Electrodiagnostic medicine. Amsterdam: Elsevier, 2002:1-1524.

5 Shapiro, BE, Preston DC. Electromyography and neuromuscular disorders. Woburn: Butterworth-Heinemann, 2005: 1-685. 